\title{
Produção para autoconsumo por agricultores familiares do município de Comodoro, região sudoeste de Mato Grosso
}

A produção destinada ao autoconsumo tem sido uma estratégia recorrente de agricultores familiares, por ser fundamental para a reprodução social do segmento apesar de ser constantemente caracterizadas equivocadamente como produções residuais (sem qualidade). Neste sentido, nesta pesquisa objetivou-se verificar a contribuição da produção destinada ao autoconsumo para os agricultores familiares do município de Comodoro-MT. Foram investigados os agricultores familiares pertencentes a quatro associações ativas no Conselho Municipal de Desenvolvimento Rural Sustentável. A amostra da população foi realizada através de amostragem sistemática por comunidade/localidade, resultando em um total de 97 agricultores. Foi realizada entrevista entre outubro e dezembro de 2015 com auxílio de formulário semiestruturado e os dados obtidos foram analisados por meio do software Excel e representados através de frequência absoluta e relativa. Todos os agricultores produzem para o autoconsumo, por conta da garantia da soberania alimentar da família. Relativo aos produtos de origem vegetal destacase a produção de olerícolas e os de origem animal os ovos são os mais consumidos pelas famílias. Nas unidades de produção que apresentam pouca diversificação de produtos de origem vegetal e animal a família tem alimentação restritiva, quando comparadas as unidades diversificadas. Os agricultores consideram suficiente sua produção de autoconsumo, com exceção da produção de suínos. A escolha das olerícolas e frutíferas a serem produzidas dependem dos hábitos alimentares de cada família, sendo neste caso, o autoconsumo decisivo na variedade de vegetais cultivados nas unidades de produção. Conclui-se que é de suma importância a produção de autoconsumo para os agricultores familiares de Comodoro-MT, havendo necessidade de fomento de propostas que contribuam com a redução dos problemas associados ao processo de modernização, insegurança alimentar e a perda de raízes culturais.

Palavras-chave: Soberania Alimentar; Olericultura; Fruticultura; Diversificação da Produção.

\section{Production for self-consumption by family farmers in the municipality of Comodoro, sw Mato Grosso state}

\begin{abstract}
The production destined to self-consumption has been a recurrent strategy of family farming, because it is of fundamental importance for the social reproduction of that segment, in spite of being mistakenly characterized as residual productions (without quality). In this sense, this study aimed to verify the contribution of production destined to self-consumption for the family farmers of Comodoro (Mato Grosso State) municipality. We investigated the family farmers belonging to four active associations of the Municipal Council for Sustainable Rural Development. The population sample was carried out by community/locality resulting in totally 97 farmers. An interview was done between October and December 2015, with the help of a semi-structured form and the data obtained was analyzed using Excel software and represented by absolute and relative frequency. All farmers produce for self-consumption because of the guarantee for food sovereignty. Referring to products of vegetal origin, the olive oil production and those of animal origin such as the eggs are mostly consumed by the families. In the production units with low diversification of plant and animal products, the family has a restricted diet, compared to the diversified units. The farmers consider its production sufficient for self-consumption, except for the pig production. The choice for olive and fruit trees production depends on the dietary habits of each family, and in this case the self-consumption is decisive on the variety of vegetables planted in the production units. It is concluded that the production for self-consumption is extremely important for the family farmers of Comodoro - Mato Grosso State, and that there is a need to promote proposals which contribute to the reduction of problems associated to the modernization process, food insecurity and the loss of cultural roots.
\end{abstract}

Keywords: Food Sovereignty; Vegetable Crops; Diversification of Production.

Topic: Química Agrícola e Ambiental

Reviewed anonymously in the process of blind peer.

Keller Regina Soares

Universidade do Estado de Mato Grosso, Brasil http://lattes.cnpq.br/4202337628291300

kelleragronomia@hotmail.com

Esvanio Edipo da Silva Ferreira

Universidade Federal de Mato Grosso, Brasil

http://lattes.cnpq.br/8821951727659938

esvanioedipo@hotmail.com

\section{Santino Seabra Junior}

Universidade Federal de Mato Grosso, Brasil

http://lattes.cnpq.br/4990974747534079

santinoseabra@unemat.br

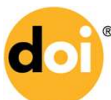

DOI: 10.6008/SPC2179-6858.2017.001.0003
Received: 19/04/2016

Approved: 21/05/2016

Sandra Mara Alves da Silva Neves

Universidade Federal de Mato Grosso, Brasil

http://lattes.cnpq.br/6430066425008976

ssneves@unemat.br

Leandro Batista da Silva

Universidade do Estado de Mato Grosso, Brasil

http://lattes.cnpq.br/2562361584173993

agrosilva.mt@gmail.com

Referencing this:

SOARES, K. R.; FERREIRA, E. E. S.; SEABRA JUNIOR, S.; NEVES, S. M. A. S.; SILVA, L. B.. Produção para autoconsumo por agricultores familiares do município de Comodoro, região sudoeste de Mato Grosso. Revista Ibero-Americana de Ciências Ambientais, v.8, n.1, p.29-39, 2017. DOI: http://doi.org/10.6008/SPC2179-

6858.2017 .001 .0003 


\section{INTRODUÇÃO}

O autoconsumo produzido pela agricultura familiar possui raízes profundas no campesinato, sendo que muitos valores e práticas tradicionais ao modo de vida camponesa permanecem e são recorrentes no âmbito da agricultura familiar (GRISA, 2007). Nesse sentido Wanderley (2003) compreende que o processo de transformação do campesinato para a agricultura familiar não deve ser considerado como passagem de uma situação de isolamento social e de exclusão de mercado para outra de integração econômica e social no conjunto da sociedade.

Nesse sentido, Schneider et al. (2008) consideram que existem elementos comuns entre os camponeses e agricultores familiares dentre os quais se destaca as noções de trabalho, produção e família que operam de forma unificada e sistêmica. De acordo com Grisa (2007), a produção para autoconsumo é uma estratégia adotadas tanto em situações que os agricultores podem ser considerados familiares ou camponeses.

Estudos realizados por Wedig (2009) demonstram o quanto a produção para autoconsumo é importante para a população rural, pois segundo a autora essa produção ocupa um lugar fundamental nas famílias de agricultores, através da garantia de boa parte da alimentação da família, atendendo não somente as necessidades fisiológicas, mas também as demandas culturais e aos hábitos alimentares da família. Nessa perspectiva, Grisa (2007) aponta que a produção de autoconsumo é uma fonte de reprodução material e cultural das famílias de agricultores, que passam a estar menos expostos a mercantilização além de propiciar a geração de renda não monetária a essas famílias.

A produção destinada ao autoconsumo é fundamental para a reprodução social de propriedades familiares, entretanto o desenvolvimento dessa atividade pela agricultura familiar deixa essas unidades produtivas caracterizadas como atrasadas ou antieconômicas (GAZOLLA, 2006). Segundo o autor, estudiosos da temática por muito tempo acreditaram na interferência da produção de alimento para o consumo próprio, na escolha das atividades e seu desenvolvimento principalmente aquelas que visam à produção em grande escala e o lucro a qualquer custo.

Por conta de sua importância no abastecimento alimentar desses agricultores e pela eventual geração de renda extra para essas famílias, a produção visando o autoconsumo é uma estratégia recorrente entre os agricultores familiares, sendo primordial para o estabelecimento destes no campo, por propiciar soberania alimentar e de certa forma reduzir os impactos da pobreza rural. De acordo com Gazolla e Schneider (2005), a produção para o uso e consumo próprio de alimentos, bens e meios de trabalho, continua a ser uma marca características da agricultura familiar.

A produção para autoconsumo, inúmeras vezes é equivocadamente caracterizada como residual e associada a pequenas quantidades ou produtos sem qualidade para padrões de mercado, quando deveria qualificar o seu valor de uso para os agricultores familiares. No entanto, é notória a importância dessa produção, que pode gerar a autonomia produtiva e reprodutiva do grupo doméstico, pois é por meio da produção para o autoconsumo que o agricultor familiar não depende, totalmente do ambiente social e 
econômico em que está inserido e, principalmente, não depende das constantes flutuações das condições de troca no mercado (ANJOS et al., 2003).

Nesse sentido, neste trabalho objetivou-se verificar a importância que a produção destinada ao autoconsumo tem para os agricultores familiares do município de Comodoro (MT), considerando a sua função no processo de reprodução social, e na diversificação produtiva e econômica.

\section{METODOLOGIA}

\section{Área de Estudo}

O município de Comodoro está situado na região sudoeste mato-grossense de planejamento (Figura 01), possui área de unidade territorial correspondente a $21.589,294 \mathrm{~m}^{2}$, com população estimada em 19.533 habitantes, com densidade demográfica de $0,84 \mathrm{hab} / \mathrm{km}^{2}$ (IBGE, 2010).

O clima do município é do tipo Equatorial quente-úmido, dominado pela Massa Equatorial Continental, com uma temperatura média anual que varia de $25^{\circ} \mathrm{C}$, chuvas concentradas no verão (outubro a abril) e um período seco bem definido, durante a estação de inverno (maio a setembro) (MATO GROSSO, 2010).

Segundo o censo do Instituto Brasileiro de Geografia e Estatística, o agronegócio passa a ter importância na economia de Comodoro a partir do declínio do ciclo da madeira, e em 2010 ocupava uma área de aproximadamente 300 mil ha com lavouras e pastagens. No município a produção de soja é a principal atividade agrícola desenvolvida, ocupando uma área em torno de $40 \mathrm{mil}$ ha, enquanto que as plantações de arroz, milho, feijão e café ocupavam uma área de 10 mil ha em 2007 (IBGE, 2010).

No município, existem agricultores assentados em 11 projetos de assentamento (PAs) e a agricultura familiar, sendo estes os maiores responsáveis pela produção de alimentos no município, onde a diversidade produtiva é uma relevante característica (OPAN, 2014).

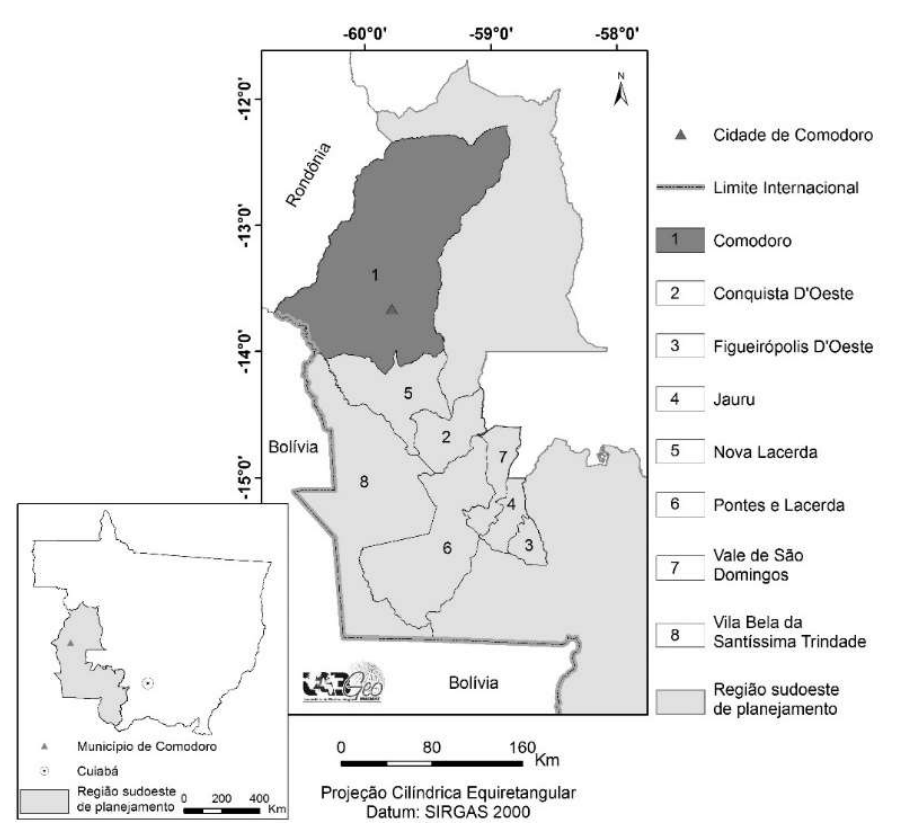

Figura 01: Comodoro no contexto da região Sudoeste de planejamento do estado de Mato Grosso, e municipal. Fonte: LabGeo UNEMAT (2016). 


\section{Procedimentos Metodológicos}

Para a realização desta pesquisa foi adotada a metodologia quanti-qualitativa para a compreensão dos fenômenos diários reais baseados nas informações obtidas durante a realização de entrevistas e das conversas informais mantidas com os agricultores durante o estudo. Conforme apontamentos de Minayo (1974), a utilização do método quali-quantitativo proporciona entender o ator social como gente, considerando sua condição social, suas crenças, valores e significados.

Os agricultores familiares foram escolhidos segundo o método de amostragem sistemática por comunidade/localidade, onde o erro amostral pode ser aproximado ao da amostragem aleatória simples, garantindo a representatividade na pesquisa, uma vez que todas as associações entrassem na amostra (MATTAR, 1999). Assim, o sorteio da família entrevistada ocorre em todas as associações.

O tamanho da amostra foi determinado conforme proposição de Thompson (1992), em que é possível estimar essa amostra proporcionando um grau de confiabilidade elevada utilizando a seguinte fórmula:

$$
\begin{array}{r}
n=\frac{1}{\left(\frac{r^{2}}{Z^{2} \cdot y^{2}}+\frac{1}{N}\right)} \\
\mathrm{Onde}, \\
\mathrm{n}=\text { número de amostras; } \\
\mathrm{r}=\text { margem de erro de uma variável significativa da população; } \\
\mathrm{Z}=\text { grau de confiança (95\%); } \\
\mathrm{y}=\text { desvio padrão de uma variável significativa da população; } \\
\mathrm{N}=\text { número total de indivíduos da população considerada. }
\end{array}
$$

Com o estabelecimento da amostra procedeu-se a coleta de dados, ocorrida entre os meses de outubro e dezembro de 2015. Para a realização das entrevistas foi utilizado um formulário semiestruturado aplicado ao agricultor (a) responsável pela unidade de produção familiar. 0 formulário foi composto por 80 questões abertas e fechadas, com abrangência na caracterização social e produtiva, contemplando a produção para autoconsumo. Dois eixos nortearam a pesquisa e foram focos nos questionários: a estrutura produtiva e o capital social.

As entrevistas foram realizadas com os agricultores familiares vinculados as seguintes associações: 1. Associações dos Produtores Rurais da Gleba Gavião Real; 2. Associação de Pequenos Produtores Rurais da Noroagro; 3. Associação dos Pequenos Produtores Rurais Unidos do Limão; e 4. Associação dos Pequenos Agricultores Rurais 25 de julho da Miranda Estância II, contabilizando ao todo 97 agricultores familiares, distribuídos entre as quatro Associações da seguinte forma: 37, 25, 18 e 17 agricultores, respectivamente.

O município conta com pelo menos 12 associações de agricultores familiares. A escolha das quatro associações se deu em virtude destes agricultores se encontrarem atuantes com o Conselho Municipal de Desenvolvimento Rural Sustentável (CMDRS), ativos quanto à documentação da entidade e quanto a organização dos agricultores e principalmente pelo fato destes realizarem produção voltada ao autoconsumo dessas famílias. 
Para quantificar a diversidade da renda, foi utilizado o índice de diversidade de Simpson (Simpson index of diversity - SID) (Simpson, 1949). Este índice foi escolhido porque leva em conta toda a variedade de fontes de renda no estabelecimento, considerando o quanto cada fonte de renda contribui para a renda onfarm total do produtor. A renda foi dada pelo VBP declarado na DAP para cada produto específico. Aplicouse a seguinte equação:

$$
S I D=1-\sum_{i=1}^{N}\left(\frac{X_{i}}{\sum_{t=1}^{N} X_{i}}\right)^{2}
$$

Onde: $\mathrm{Xi}=\mathrm{VBP}$ de cada produto; e $\mathrm{N}=$ número de produtos.

A partir do resultado dessa equação, assume-se que o valor 0 representa a unidade produtiva com a exploração de apenas uma espécie, e se aproxima de 1 à medida que ocorre o aumento da diversidade de espécies 'produzidas nas propriedades. Dessa maneira a diversificação será definida a partir das seguintes categorias: muito especializado $(S I D=0)$, especializado ( $S I D>0,0$ e menor que $\leq 0,35$ ), diversificado $(S I D>0,35$ e $\leq 0,65$ ), e muito diversificado (SID >0,65), conforme proposto por Sambuichi et al. (2014).

As perguntas dos questionários geraram o banco de dados, cujas respostas foram sistematizadas em planilha Excel e são representadas por meio de estatísticas de frequência absoluta (Fi), que indica quantas vezes a opção foi selecionada pelos entrevistados, e a frequência relativa (Fri), que corresponde à razão entre a frequência absoluta e o número total de observações, expressa em percentual.

\section{RESULTADOS E DISCUSSÃO}

Em todas as propriedades investigadas foi observada a prática da produção para autoconsumo, sendo está uma estratégia utilizada pelos agricultores familiares para a redução de gastos com aquisição de gêneros alimentares provindos de fora da propriedade, onde os atores sociais salientam a economia gerada por não precisar comprar determinados tipos de alimentos.

Esses dados são facilmente explicados, pois conforme apontamentos de Santos e Barreto (2005) a valorização do autoconsumo reduz as despesas com a sua alimentação, além de tê-la disponível em maior quantidade e qualidade. Ao adotar essa forma de produção, diversificada e voltado ao autoconsumo, tem permitido a reprodução familiar do agricultor dentro do espaço agrícola, garantindo assim sua soberania alimentar (PELINSKI et al. 2006).

De forma prática, o cálculo do valor de autoconsumo, pode ser realizada pela contabilização da redução de despesas ou pela atribuição de valor de comercialização destes produtos consumidos, caso fossem vendidos. Optar pela atribuição do valor de venda e não o preço de compra se deve principalmente pela variação de preços de compra (ANJOS et al., 2004)

Além disso, conforme afirmação de Anjos et al. (2003), a importância do autoconsumo reflete no comportamento da renda agrícola e, consequentemente, na renda total das explorações. Este fato demonstra de modo inequívoco a importância que pode assumir esta dimensão do processo de reprodução familiar. Essa produção possui, também, outro papel fundamental na reprodução da agricultura familiar, pois 
para Ellis (2000) permite que as formas familiares de produção e trabalho sobrevivam e se reproduzam em uma sociedade capitalista cada vez mais competitiva.

O acesso total ou parcial aos alimentos pode ser garantido pela produção realizada para o autoconsumo, produção esta muito comum na agricultura familiar (DUTRA, 2013). No entanto segundo o autor, a falta de acesso a terra impede a existência desta produção. Quando em condições de ser realizada a produção para autoconsumo possibilita o acesso direto aos alimentos, pois estes seguem da unidade de produção (lavoura) para a unidade de consumo (casa), sem nenhum processo de intermediação que a torne valor de troca (GRISA, 2010).

A agricultura familiar representa a ocupação social mais equitativa do espaço agrário favorecendo a valorização das dimensões social, ambiental e cultural da produção para autoconsumo, e propicia acesso direto aos alimentos, o que caracteriza a segurança alimentar e nutricional (CAPORAL et al., 2006; VOGT et al., 2007).

Outra justificativa dada pelos agricultores familiares de Comodoro (MT) para a produção de autoconsumo está associada com a questão da soberania alimentar, pois para 92\% das famílias entrevistadas, além da redução de gastos com a aquisição de alimentos, o autoconsumo é importante, pois garante aos agricultores e família diversidade de alimentos disponíveis em sua alimentação diária, o que não seria possível se todos esses alimentos tivessem que ser adquiridos fora da unidade produtiva.

Conforme Maluf et al. (2005), no âmbito da agricultura familiar, a possibilidade de produção de autoconsumo é apresentado como uma das formas para garantir a segurança alimentar em áreas rurais, e acrescentam que a produção para autoconsumo contribui para a disponibilização de diversidade alimentar para os produtores rurais. Ellis (2000) corrobora com o exposto ao relatar que fortalecer a produção de autoconsumo resulta na diversificação das estratégias de vivência e garante segurança alimentar aos agricultores familiares pela redução da vulnerabilidade em que geralmente se encontram.

Também é apontada como contribuição da produção para autoconsumo a questão relativa à qualidade dos produtos, uma vez que $96 \%$ dos agricultores relataram que se sentem mais seguro cultivando o próprio alimento, geralmente livres de agrotóxicos.

Geralmente, alimentos produzidos por agricultores familiares e destinados ao autoconsumo, são normalmente isentos de agrotóxicos e outros produtos químicos, em razão da escala de produção, minimizando os riscos desta contaminação e os relacionados ao consumo de alimentos industrializados, contribuindo para a segurança alimentar e nutricional de famílias da zona rural (GRISA, 2009).

De acordo com o conceito construído pelo Conselho Nacional de Segurança Alimentar e Nutricional (CONSEA), uma alimentação adequada e saudável é um "direito humano básico, com a garantia ao acesso permanente e regular, de forma socialmente justa, a uma prática alimentar adequada aos aspectos biológicos e sociais dos indivíduos, de acordo com o ciclo de vida e as necessidades alimentares especiais, pautada no referencial tradicional local. Deve atender aos princípios da variedade, equilíbrio, moderação, prazer (sabor), dimensões de gênero e etnia, e formas de produção ambientalmente sustentáveis, livre de contaminantes 
físicos, químicos e biológicos e organismos geneticamente modificados. Este conceito explicita a perspectiva de uma alimentação livre de alimentos com agrotóxicos e transgênicos" (BRASIL, 2007).

Apesar da produção para autoconsumo estar presente em 100\% das unidades de produção familiar, essa prática não ocorre na mesma intensidade em todas as unidades. Dessa maneira, em $10 \%$ das unidades de produção foi verificada que a intensidade de produção para autoconsumo está ligada a base tecnológica da agricultura e condições técnicas da produção agrícola, enquanto que observou que em $22 \%$ dessas unidades a proporção dessa produção se deve a proximidade aos mercados, o que possibilita os agricultores a terem acesso a compra de alimentos de forma que isso interfira diretamente na forma que estes desenvolvam essa produção de autoprovisionamento. Em $68 \%$ das unidades produtivas, foi possível verificar que a intensidade da produção de autoconsumo está ligada a fatores culturais, ou seja, essa produção é realizada de acordo com os conhecimentos tradicionais intrínsecos a essa população, onde este hábito pela prática histórica é uma influência determinante na realização ou não deste tipo de produção.

Conforme constatação feita por Wagner et al. (2007), a produção para autoconsumo está imbricada num complexo conjunto de elementos que influenciam na decisão de produzir para o consumo familiar, como a força de trabalho disponível, condições técnicas de produção, os preços da alimentação nos mercados, a cultura e a motivação pessoal para a produção.

Pelinsk et al. (2006), afirmam que o autoconsumo oferece segurança alimentar aos agricultores, garantindo-Ihes acesso a alimentos básicos e de qualidade e quantidade suficiente, indicando a sustentabilidade maior do agricultor e garantindo-lhes a sobrevivência no meio rural.

Os agricultores familiares têm produzido para o autoconsumo familiar alimentos de origem vegetal e animal (Tabela 1). A respeito dos alimentos de origem vegetal, produzidos para o autoconsumo em suas unidades produtivas, observou-se que a produção de olerícolas (horta) é uma das estratégias mais utilizadas por esses agricultores, seguida da produção de frutíferas.

Segundo Gazolla (2004), a presença generalizada da produção para o autoconsumo entre os agricultores familiares aponta a importância da horta e do pomar nos estabelecimentos pesquisados. $O$ autor encontrou que a maioria dos estabelecimentos familiares de Alto Uruguai preza por possuir horta $(92,1 \%)$ e pomar (89,9\%), e não há discrepâncias entre os universos pesquisados.

Tabela 1: Atividades desenvolvidas pelos agricultores familiares de Comodoro (MT) com a finalidade de autoconsumo.

\begin{tabular}{|c|c|c|}
\hline Produção & $\mathrm{F}_{\mathrm{i}}$ & $\mathrm{F}_{\mathrm{ri}}(\%)$ \\
\hline Ovos & 95 & 21,30 \\
\hline Olerícolas (Horta) & 85 & 19,06 \\
\hline Frutíferas (Pomar) & 81 & 18,16 \\
\hline Leite & 76 & 17,04 \\
\hline Frango/Galinha & 65 & 14,57 \\
\hline Suíno & 44 & 9,87 \\
\hline Total & 446 & 100 \\
\hline
\end{tabular}

$\mathrm{Fi}=$ Frequência absoluta; Fri= Frequência relativa.

A produção de alimentos de origem animal, além de serem utilizados como fonte de proteína na alimentação dos agricultores também é destinada à comercialização. A produção de ovos é a atividade 
desenvolvida em um maior número de unidades produtivas, destinada ao autoconsumo. Os ovos são consumidos na forma de gemada, frito, cozido e também muito utilizados na fabricação de bolos, bolachas, biscoitos, pães, etc.

A pecuária leiteira é a segunda atividade mais desenvolvida pelos agricultores familiares de Comodoro. O leite além de ser consumido de forma in natura por essas famílias também utilizam esse produto na forma de derivados (queijo, requeijão, coalhada e doce) e a produção de ovos foi à atividade desenvolvida em um maior número de unidades produtivas destinada ao autoconsumo, aparecendo em uma frequência de 95 respostas. Posteriormente, os agricultores apontam as atividades de produção de frango e suíno, como de grande relevância para o consumo familiar e comercialização.

No entanto, ressalta-se que os entrevistados que não possui diversificação na produção vegetal e animal, destinados ao autoconsumo, tem uma alimentação um tanto quanto restritiva em relação as demais famílias, sendo necessária a aquisição dos alimentos não produzidos de outros agricultores.

Segundo Ellis (2000) os determinantes da diversificação dos meios de vida rurais são variados e podem estar relacionados a aspectos edafoclimáticos ou socioeconômicos que se manifestam através da sazonalidade, dos riscos, da vulnerabilidade, das migrações, dos efeitos do mercado de trabalho, do aceso ao crédito e a outros ativos. Por sua vez, os efeitos da diversificação se apresentam ou se expressam como atributos da diversidade na forma de atividades (pluriatividade) e de rendimentos (multirendimentos), de tal forma que podem ser medidos ou classificados segundos critérios quantitativos e qualitativos.

Quanto à suficiência da produção para autoconsumo, utilizou-se como referência a resposta dos agricultores entrevistados, em que a maioria salienta que a produção vegetal e animal existente é o suficiente para suprir as necessidades de alimentação em quantidade e qualidade da família (Tabela 2), entretanto constatou-se um percentual relevante de investigados que necessitam adquirir os alimentos para complementar sua alimentação.

Tabela 2: Percentual de suficiência dos produtos provindos da horta e pomar, e as produções de frango, suínos e ovos são suficientes para suprir as necessidades alimentares das famílias.

\begin{tabular}{lcccccc}
\hline & Horta (\%) & Pomar (\%) & Frango (\%) & Suínos (\%) & Ovos (\%) & Bovinos (\%) \\
\hline Sim & 87,63 & 74,86 & 85 & 44 & 97 & 2 \\
Não & 12,37 & 25,14 & 15 & 56 & 3 & 98 \\
\hline Total & 100 & 100 & 100 & 100 & 100 & 100 \\
\hline
\end{tabular}

Fi= Frequência absoluta; Fri= Frequência relativa

A produção de ovo caipira, os produtos da horta, frango e as frutíferas foram às atividades que apresentaram maior percentual de suficiência na alimentação desses agricultores familiares, atendendo as necessidades de consumo da família, não havendo necessidade da aquisição destes alimentos em outros locais fora da unidade de produção. Conforme observado, esses alimentos têm feito parte da base diária alimentar desses agricultores, o que de certa forma tem colaborado para a soberania alimentar dessas famílias.

Isso corrobora com a afirmação de Gazolla (2004), ao expor que grande percentagem de pessoas que possuem horta e pomar e que estas cobrem as necessidades de consumo das famílias. Segundo o autor, os 
agricultores continuam valorizando e produzindo os alimentos provenientes da horta e do pomar para a sua alimentação e que a mercantilização ainda não atingiu esse tipo de produção provindo da agricultura familiar.

A alta percentagem de insuficiência da produção de suínos chama atenção, onde mais da metade dos agricultores citam sua produção como insuficiente para atender a demanda de consumo familiar. Essa situação pode ser explicada pelo fato de que grande parte da produção de suínos é destinada a venda, sendo estes animais consumidos pelos agricultores apenas em ocasiões especiais. Conforme afirmação de Grisa (2007), a insuficiência pode ser atribuída a preferência por produtos forâneos, limitação de área, opção da família pela venda do produto ou de pôr não produzir etc.

A escolha das olerícolas e frutíferas a serem produzidas dependem dos hábitos alimentares de cada família, sendo neste caso, o autoconsumo, decisivo na variedade de vegetais cultivados nas unidades de produção. As principais olerícolas produzidas pelos agricultores familiares pesquisados são: mandioca (79\%), melancia (70\%), alface (52\%), melão (50\%), Abacaxi $(50 \%)$ cebola de folha (49\%), couve (41\%), quiabo (40\%), jiló (37\%), maxixe (36\%), cachi (35\%), abóbora menina brasileira e goianinha (35\%), rúcula (27\%), batata doce (22\%), salsinha (18\%), pimenta bode (12\%), vagem (11\%), pimentão (11\%).

Esses dados encontrados sobre a variedade de olerícolas e frutíferas cultivadas em Comodoro-MT corrobora com os dados encontrados por Queiroz (2014), em seus estudos com agricultores familiares na mesma região do estado de Mato Grosso, em que a autora encontrou as seguintes hortícolas produzidas: abóbora, mandioca, pepino, melancia, melão, jiló, berinjela, cenoura, coentro, alface, almeirão, rúcula, milho, cebolinha, salsinha, abobrinha, beterraba, batata doce, vagem, pimentão, milho verde, tomate cereja, maxixe, couve, pimenta, rabanete, pimenta doce, yura, couve-chinesa e cachi.

Abreu e Watanabe (2008) relataram que agricultores familiares de Ouro Preto D'Oeste-RO, apresentam sistemas de produção diversificados, cultivando em suas propriedades olerícolas e pomares caseiros altamente diversificados, que são responsáveis por fornecer as famílias alimentação diversificadas.

\section{CONCLUSÕES}

Constatou-se que a produção para autoconsumo tem sido uma prática adotada pelos agricultores familiares do município de Comodoro/MT, e tem contribuído para a soberania alimentar dessas famílias. Além disso, foi possível verificar que os principais fatores para a produção de alimentos destinados ao autoconsumo, é a soberania alimentar, a geração de renda não monetária obtida pela redução nos gastos com aquisição de alimentos, e a qualidade dos produtos referentes à ausência de agrotóxicos.

A produção diversificada tem possibilitado a soberania alimentar desses agricultores através da disponibilidade básica de alimentos. A criação galinhas poedeiras, frango, suínos e bovinos assim como a presença de hortas e pomares têm contribuído para a oferta de alimentos em quantidade satisfatória, com consequente redução de despesas. 


\section{AGRADECIMENTOS}

Ao projeto de extensão: “Núcleo de extensão em Desenvolvimento Territorial da grande Cáceres: uma estratégia de desenvolvimento sustentável por meio de ações que fortaleçam a produção agroecológica, agroindustrialização, comercialização e a atuação das mulheres" financiado no âmbito do Edital CNPq/MDA/ SPM n $n^{\circ}$ 11/2014 - Apoio a implantação e manutenção de núcleos de extensão em desenvolvimento territorial. À Coordenação de Apoio ao Pessoal de Nível Superior - Capes pela concessão de bolsa de mestrado a autora deste texto.

\section{REFERÊNCIAS}

ABREU, L. S.; WATANABE, M. A.. Agroforestry systems and food security among smallholder farmers of the Brazilian Amazon: A strategy for environmental global crisis. In: ORGANIC WORLD CONGRESS. Proceedings. Modena: IFOAM; ISOFAR, 2008. p.472-475

ANJOS, F. S.; SCHNEIDER, S.. Abrindo a caixa verde: estudo sobre a importância do autoconsumo na agricultura familiar gaúcha. In: CONGRESSO BRASILEIRO DE ECONOMIA E SOCIOLOGIA RURAL, 42. Cuiabá. Anais. Cuiabá: SOBER. 2004.

ANJOS, F. S.; SCHNEIDER, S.. Agricultura familiar, desenvolvimento local e pluriatividade no Rio Grande do Sul: a emergência de uma nova ruralidade. Pelotas: PPGA/UFRGS (PGDR), 2003.

\section{BRASIL. RADAMBRASIL. Levantamentos dos Recursos} Naturais: DNPM/CPRM Projeto RADAMBRASIL - Folha SD-20 Guaporé. Rio de Janeiro: IBGE, 1979.

CAPORAL, F. R.; COSTABEBER, J. A.. Segurança alimentar e agricultura sustentável: uma perspectiva agroecológica. Rev. Bras. de Agroecologia, v.1, n.1, 2006.

DUTRA, L. V.. Insegurança alimentar e nutricional e produção para autoconsumo na ona rural de São Miguel do Anta, Minas Gerais. Dissertação (Mestrado em Agrocologia) - Universidade Federal de Viçosa, Viçosa, 2013.

ELLIS, F.. Rural livelihoods and diversity in developing countries. Oxford: Oxford University Press, 2000.

GAZOLLA, M.. Agricultura familiar, segurança alimentar e políticas públicas: Uma análise a partir da produção para autoconsumo no território do Alto Uruguai/RS. Dissertação (Mestrado em Desenvolvimento Rural) - Universidade Federal do Rio Grande do Sul, Porto Alegre, 2004.

GAZOLLA, M.. O processo de mercantilização do consumo de alimentos na agricultura familiar. In: SCHNEIDER, S.. A diversidade da agricultura familiar. Porto Alegre: EdUFRGS, 2006. p.82-103

GAZOLLA, M.; SCHNEIDER, S.. Produção para autoconsumo e segurança alimentar: uma abordagem com base na agricultura familiar. In: CONGRESSO BRASILEIRO DE SOCIOLOGIA, 12. Anais. Belo Horizonte: Sociedade Brasileira de Sociologia, 2005. p.01-21.
GRISA, C.. A produção "pro gasto": um estudo comparativo do autoconsumo no Rio Grande do Sul. Dissertação (Mestrado em Desenvolvimento Rural) - Universidade Federal do Rio Grande do Sul, Porto Alegre, 2007.

GRISA, C. A.. Produção para autoconsumo na agricultura familiar: uma análise a partir da teoria da sociedade de risco. Revista Varia Scientia, v.8, n.14, p.171-200, 2009.

GRISA, C.. Diferentes olhares na análise de políticas públicas: considerações sobre o papel do Estado, das instituições, das ideias e dos atores sociais. Sociedade e Desenvolvimento Rural, v.4, n.1, 2010.

IBGE. Instituto Brasileiro de Geografia e Estatístico. Censo Demográfico 2010. Rio de Janeiro: IBGE, 2010.

MALUF, R. S; REIS, M. C.. Manifestação de Insegurança Alimentar. REDECAPA, 2005.

MATO GROSSO. Secretaria de Estado e Planejamento e Coordenação Geral. Anuário Estatístico de Mato Grosso. Cuiabá: SEPLAN, 2010.

MATTAR, F. N.. Pesquisa de marketing. 5 ed. São Paulo: Atlas,1999.

MINAYO, M. C. S.. Pesquisa social: teoria método e criatividade. 16 ed. Petrópolis: Vozes, 2000.

OPERAÇÃO AMAZÔNIA NATIVA. ICMS ecológico: oportunidade para o desenvolvimento municipal em Mato Grosso. Cuiabá: OPAN, 2014.

PELINSKI, A.; AHRENS, D. C.; MILLEO, R. D. S.; ZEMKE, E. W.; BERNARDI, D. A.; RITCHER, A. S.. Autoconsumo: sua relevância na sustentabilidade da agricultura agroecológica. In: CONGRESSO BRASILEIRO DE AGROECOLOGIA, 4. Anais. Belo Horizonte, 2006.

QUEIROZ, R. F. N.. Análise agroclimática do melão na região sudoeste mato-grossense: contribuições para o fortalecimento da agricultura familiar. Dissertação (Mestrado em Ambiente e Sistemas de Produção Agrícola) Universidade do Estado de Mato Grosso, Tangará da Serra, 2014.

SAMBUICHI, R. H. R.; OLIVEIRA, M. A. C.. Análise das linhas de crédito do Pronaf para o desenvolvimento sustentável da 
agricultura familiar. Cadernos de Agroecologia, v.6, n.2, p.11-186, 2011.

SANTOS, J. A.; BARRETO, R.. Agricultoras descobrem nova forma de gerar renda e garantir uma alimentação segura. Revista Agriculturas, Rio de Janeiro, v.2, n.3, p.31-33, 2005.

SCHNEIDER, S.; NIEDERLE, P. A.. Agricultura familiar e teoria social: a diversidade das formas familiares na agricultura. In: FALEIRO, F. G.; FARIAS NETO, A. L.. Savanas: desafios e estratégias para o equilíbrio entre sociedade, agronegócio e recursos naturais. Planaltina: Embrapa Cerrados, 2008. p.989-1014

SIMPSON, E. H.. Measurement of diversity. Nature, v.163, p.688, 1949 .

THOMPSON, S. K.. Sampling: Wiley series in probably and mathematical statistics. New York: John Wiley \& Sons, 1992

VOGT, S. P. C., SOUZA, R. S.. Políticas públicas e segurança alimentar: estudo de caso do programa de compra antecipada especial com doação simultânea (fome zero) no município de tenente Portela-RS. In: CONGRESSO BRASILEIRO DE SISTEMAS DE PRODUÇÃO, 7. Anais. Londrina: 2007.

WAGNER, S. A.; MARQUES, F. C.; MENASCHE, R.. Agricultura familiar a mesa. In: MENASCHE, R.. A agricultura familiar à mesa: Saberes e práticas da alimentação no Vale do Taquari. Porto Alegre: EdUFRGS, 2007.

WANDERLEY, M. N.. Agricultura familiar e campesinato: rupturas e continuidade. Estudos Sociedade e Agricultura, n.21, 2003.

WEDIG, J. C.. Agricultoras e Agricultores a mesa: um estudo sobre campesinato e gênero a partir da antropologia da alimentação. Dissertação (Mestrado em Desenvolvimento Rural) - Universidade Federal do Rio Grande do Sul, Porto Alegre, 2009. 